\title{
Generalized linear-scaling localized-density-matrix method
}

\author{
WanZhen Liang, Satoshi Yokojima, and GuanHua Chen \\ Department of Chemistry, The University of Hong Kong, Pokfulam Road, Hong Kong
}

(Received 12 June 1998; accepted 23 October 1998)

\begin{abstract}
A generalized linear scaling localized-density-matrix (LDM) method is developed to adopt the nonorthonormal basis set and retain full Coulomb differential overlap integrals. To examine its validity, the method is employed to evaluate the absorption spectra of polyacetylene oligomers containing up to 500 carbon atoms. The semiempirical Hamiltonian for the $\pi$ electrons includes explicitly the overlap integrals among the $\pi$ orbitals, and is determined from the ab initio HartreeFock reduced single-electron density matrix. Implementation of the generalized LDM method at the ab initio molecular orbital calculation level is discussed. () 1999 American Institute of Physics. [S0021-9606(99)31104-1]
\end{abstract}

\section{INTRODUCTION}

There is a growing interest in numerical evaluation of the electronic structures of complex and large systems like proteins, aggregates and nanostructures. Ab initio and semiempirical molecular orbital calculations are usually limited to small and medium size molecular systems. The obstacle lies in rapidly increasing computational costs as the systems become larger and more complex. The computational time $t_{\mathrm{CPU}}$ is proportional to a certain power of the system size, i.e., $t_{\mathrm{CPU}} \propto N^{x}$, where $N$ is the number of electrons, and $x$ is an exponent which is usually larger than 1 . For instance, the computational time of ab initio Hartree-Fock (HF) molecular orbital calculation has an $O\left(N^{3 \sim 4}\right)$ scaling, i.e., $x=3$ $\sim 4$. To determine the electronic structures of very large systems, it is essential that the computational cost scales linearly with $N$. Several $O(N)$ methods have been developed to calculate the electronic ground state. ${ }^{1-23}$ The physical basis of these methods is "the nearsightedness of equilibrium systems." 24 The excited states of very large electronic systems are much more difficult to determine. Several linear scaling calculations based on the noninteracting electron models have been carried out to determine the excited state properties of large systems. ${ }^{10,25}$ Explicit inclusion of electronic correlation in the linear scaling calculation of the excited state properties has proven much more challenging.

A reduced single-electron density matrix $\rho$ contains important information of an electronic system. Expressed in an orthonormal basis set, the diagonal element $\rho_{i i}$ is the electron density at a local orbital $i$, and the off-diagonal element $\rho_{i j}$ $(i \neq j)$ measures the electronic coherence between two orthogonal local orbitals $i$ and $j$, where the reduced singleelectron density matrix $\rho$ is then defined as the expectation value $\rho_{i j} \equiv\left\langle\psi\left|a_{j}^{\dagger} a_{i}\right| \psi\right\rangle$ with $\psi$ being the wave function and $a_{i}^{\dagger}\left(a_{j}\right)$ the electron creation (annihilation) operator at the local orbital $i(j)$. An equation of motion (EOM) for the reduced density matrix has been solved to calculate linear and nonlinear electronic responses to external fields, ${ }^{26}$ and thus, probe the properties of the excited states. This EOM is based on the time-dependent Hartree-Fock (TDHF) approximation, ${ }^{27}$ and the computational time for solving it in the frequency domain scales as $O\left(N^{6}\right)$ while in the time domain it scales as $O\left(N^{4}\right)$. Since the calculation of the many-body wave functions is avoided, the computational effort is greatly reduced compared to the conventional sum-over-state methods. ${ }^{28-33}$ The TDHF approximation includes complete single electron excitations and some partial double, triple and other multi-electron excitations. It has been applied successfully to investigate the optical properties of conjugated polymers. ${ }^{26}$ An $O\left(N^{2}\right)$ scaling density-matrix-spectralmoment algorithm ${ }^{34}$ has been developed to calculate the envelope of the entire linear and nonlinear optical spectra of conjugated polymers containing up to 300 carbon atoms. In Ref. 35, it has been shown that the ground state off-diagonal elements $\rho_{i j}$ are negligible when the distance $r_{i j}$ between $i$ and $j$ is larger than a critical length $l_{0}$. This is a consequence of "the near-sightedness of equilibrium systems." 24 When the system is subjected to an external field $\mathbf{E}(t)$, the field induces a change $\delta \rho$ in the reduced density matrix. The induced density matrix $\delta \rho$ has a similar "near-sightedness," i.e., the off-diagonal element $\delta \rho_{i j}$ is approximately zero as the distance between $i$ and $j$ is large enough. ${ }^{35}$ Different orders of responses in $\mathbf{E}(t)$ have different critical lengths. Usually the higher the order of response $n$, the longer the critical length $l_{n}$, i.e., $l_{0}<l_{1}<l_{2}<l_{3}<\cdots$. We may truncate the $n$th order induced density matrix response $\delta \rho^{(n)}$ (note, $\delta \rho$ $\left.=\delta \rho^{(1)}+\delta \rho^{(2)}+\delta \rho^{(3)}+\cdots\right)$ by setting its elements $\delta \rho_{i j}^{(n)}$ to zero if $r_{i j}>l_{n}$. This truncation may lead to a drastic reduction of the computational time.

Recently the linear scaling localized-density-matrix (LDM) method has been developed to evaluate the properties of excited states. ${ }^{36,37}$ It is based on the TDHF approximation $^{27}$ and the above-mentioned truncation of the density matrix. Through the introduction of the critical lengths $l_{0}, l_{1}$ and others which are characteristic of the reduced density matrix, the computational time of the LDM method scales linearly with the system size $N$. The method has been tested successfully to evaluate the optical properties of conjugated polymers. ${ }^{36,37}$ In Refs. 36 and 37, the PariserParr-Pople (PPP) model $^{38}$ is adopted to describe the dynamics of $\pi$ electrons in polyacetylene (PA) oligomers. The PPP model is based on orthonormal basis set and the zero differ- 
ential overlap (ZDO) approximation for electron-electron Coulomb interaction. ${ }^{39}$ The usage of the orthonormal basis set and the ZDO approximation limit the applicability of the LDM method. $A b$ initio calculations usually use nonorthonormal basis sets (for instance, the Slater-type atomic orbitals) and include Coulomb differential overlap integrals. Most semiempirical calculations like the intermediate neglect of differential overlap (INDO) ${ }^{40}$ the modified neglect of diatomic overlap (MNDO), ${ }^{41}$ Austin Model 1 (AM1), ${ }^{42}$ and MNDO-Parametric Method 3 (PM3) ${ }^{43}$ neglect partial differential overlaps. Moreover, it has been pointed out that for conjugated polymers the differential overlap integrals should be included explicitly in order to calculate accurately both the bond orders and the optical gaps. ${ }^{44}$ Thus, it is desirable to generalize the LDM method so that the nonorthonormal basis set may be adopted and the complete Coulomb differential overlap integrals are included in the calculation. A natural choice for the nonorthonormal basis set is the atomic orbital (AO) basis set. An AO depends only on the atomic type, and is thus transferable for any atom in different molecules.

In this work we employ the AO basis set, and generalize the LDM method to calculate the excited state properties. The generalized LDM method is applied to calculate the optical absorption spectra of PA oligomers containing up to 500 carbon atoms. To simplify the calculation, we consider only the $\pi$ electrons in the systems, since these electrons are responsible for the optical spectra in the visible range. The PPP Hamiltonian is based on the orthonormal basis set. Thus, a Hamiltonian based on the nonorthonormal AO basis set is to be determined. In Sec. II an effective Hamiltonian model based on the AO basis set is proposed to describe the dynamics of $\pi$ electrons in conjugated polymers. In Sec. III the TDHF method employing the nonorthonormal basis set is developed, and its EOM is derived. In Sec. IV the LDM formalism is generalized for implementation in the nonorthonormal basis set. In Sec. V a novel algorithm is applied to PA to determine the effective Hamiltonian for the $\pi$ electrons in the nonorthonormal $\pi$ orbital basis set. In Sec. VI the absorption spectra of PA oligomers containing up to 500 carbon atoms are obtained. The linear scaling of the computational time and memory is examined in detail. The roles of different critical lengths are investigated. Further development of the LDM is discussed, and the results of this work are summarized in Sec. VII.

\section{MODEL}

A PA oligomer is a planar unsaturated organic molecule, and its valence molecular orbitals (MOs) may be divided into $\pi$ and $\sigma$ MOs. ${ }^{38}$ The $\pi$ electrons may be treated separately from the $\sigma$ electrons, and are responsible for the optical response in the optical frequency regime. The Hamiltonian for the $\pi$ electrons may be written as follows,

$$
\begin{aligned}
& H=H_{e}+H_{e e}+H_{\mathrm{ext}}, \\
& H_{e}=\sum_{i=1}^{N}\left(-\frac{1}{2} \nabla_{\mathbf{r}_{i}}^{2}+U\left(\mathbf{r}_{i}\right)\right),
\end{aligned}
$$

$$
\begin{aligned}
& H_{e e}=\sum_{i=1}^{N} \sum_{j>i} V\left(r_{i j}\right), \\
& H_{\mathrm{ext}}=\sum_{i=1}^{N} e \mathbf{E}(t) \cdot \mathbf{r}_{i},
\end{aligned}
$$

where $i$ and $j$ are, respectively, the indices of the $i$ th and $j$ th electrons, $U\left(\mathbf{r}_{i}\right)$ is the potential energy of the $i$ th electron in the field produced by the nuclei and the core and $\sigma$ electrons, $V\left(r_{i j}\right)$ is the effective Coulomb interaction between the $i$ th and $j$ th electrons with $r_{i j}$ being the distance between the two electrons, and $\mathbf{E}(t)$ is the external field. Thus, $H_{e}$ is the oneelectron part of the Hamiltonian which describes the dynamics of a single $\pi$ electron in the absence of other $\pi$ electrons. $H_{e e}$ is the two-electron part of the Hamiltonian which represents the effective Coulomb interaction among the $\pi$ electrons. $H_{\text {ext }}$ is the interaction between the $\pi$ electrons and an external electric field $\mathbf{E}(t)$.

The one-electron integral $t_{i j}$ may be expressed as

$$
t_{i j}=\left\langle\chi_{i}\left|-\frac{1}{2} \nabla_{\mathbf{r}}^{2}+U(\mathbf{r})\right| \chi_{j}\right\rangle,
$$

where $\chi_{i}$ is the $\pi \mathrm{AO}$ of the $i$ th carbon atom, and $\mathbf{r}$ is the displacement vector of an electron. Here the index $i$ represents the $i$ th carbon atom, and it increases from one end of an oligomer to the other end starting from 1. The two-electron integral $V_{i j, k l}$ may be calculated via the following expression:

$$
V_{i j, k l}=\int d \mathbf{r}_{1} d \mathbf{r}_{2} \chi_{i}^{*}\left(\mathbf{r}_{1}\right) \chi_{j}\left(\mathbf{r}_{1}\right) V\left(r_{12}\right) \chi_{k}^{*}\left(\mathbf{r}_{2}\right) \chi_{l}\left(\mathbf{r}_{2}\right) .
$$

Since the AOs are localized on individual atoms, we may keep only the diagonal terms of the one-electron integrals (i.e., $t_{i i}$ ) and the off-diagonal terms corresponding to any pairs of two orbitals that form a $\pi$ bond. In other words,

$$
t_{i j}=0,
$$

if $i \neq j$ and the $i$ th and $j$ th atoms are not bonded via a $\pi$ bond.

Unlike the $\mathrm{PPP}^{38}$ and complete neglect of differential overlap $(\mathrm{CNDO})^{45}$ methods where the differential overlap integrals are neglected, we keep all Coulomb differential overlap integrals in Eq. (6). The effective Coulomb interaction $V\left(r_{i j}\right)$ may be approximated by the Ohno formula. ${ }^{46}$ i.e.,

$$
V\left(r_{i j}\right)=\frac{U}{\sqrt{1+\left(r_{i j} / a_{0}\right)^{2}}},
$$

where $U$ is the on-site Coulomb interaction, and $a_{0}$ is a characteristic length which is approximately the bond length. Instead of evaluating Eq. (6) explicitly, two-electron integrals may be approximated by the following expression: ${ }^{47}$ 


$$
\begin{aligned}
& V_{m n, k l} \approx v_{m n, k l} S_{m n} S_{k l}, \\
& v_{m n, k l}=\frac{U}{\sqrt{1+\left(\frac{\left|\mathbf{r}_{\overline{m n}}-\mathbf{r}_{k l}\right|}{a_{0}}\right)^{2}}},
\end{aligned}
$$

where the overlap integrals $S_{i j}$ are defined as follows:

$$
S_{i j} \equiv\left\langle\chi_{i} \mid \chi_{j}\right\rangle,
$$

and $\mathbf{r}_{m n}$ is the mean displacement vector of $\mathbf{r}_{n}$ and $\mathbf{r}_{m}$, i.e.,

$$
\mathbf{r}_{m n}=\frac{1}{2}\left(\mathbf{r}_{m}+\mathbf{r}_{n}\right)
$$

\section{TDHF METHOD IN NONORTHONORMAL BASIS SET}

The EOM for the reduced single-electron density matrix $\rho$ in an orthonormal basis set has been derived within the TDHF approximation. ${ }^{26}$ Here we derive the EOM for $\rho$ in a nonorthonormal basis set. Starting with the definition of reduced single-electron density matrix $\rho\left(\mathbf{r}_{1} \theta_{1}, \mathbf{r}_{1}^{\prime} \theta_{1}^{\prime}, t\right)$ in the spin-spatial representation:

$$
\begin{aligned}
\rho\left(\mathbf{r}_{1} \theta_{1}, \mathbf{r}_{1}^{\prime} \theta_{1}^{\prime}, t\right) \\
=N \int d \mathbf{r}_{2} d \theta_{2} d \mathbf{r}_{3} d \theta_{3} \cdots d \mathbf{r}_{N} d \theta_{N} \\
\quad \times \Phi\left(\mathbf{r}_{1} \theta_{1}, \mathbf{r}_{2} \theta_{2}, \ldots, \mathbf{r}_{N} \theta_{N}, t\right) \\
\quad \times \Phi^{*}\left(\mathbf{r}_{1}^{\prime} \theta_{1}^{\prime}, \mathbf{r}_{2} \theta_{2}, \ldots, \mathbf{r}_{N} \theta_{N}, t\right),
\end{aligned}
$$

where $\Phi\left(\mathbf{r}_{1} \theta_{1}, \mathbf{r}_{2} \theta_{2}, \ldots, \mathbf{r}_{N} \theta_{N}, t\right)$ is the Slater determinant representing many-body wave function, and $\mathbf{r}_{i}$ and $\theta_{i}$ are the spatial and spin coordinate for the $i$ th electron, respectively. We write down the EOM for $\rho\left(\mathbf{r}_{1} \theta_{1}, \mathbf{r}_{1}^{\prime} \theta_{1}^{\prime}, t\right)$ :

$$
\begin{aligned}
i \hbar \dot{\rho}\left(\mathbf{r}_{1}\right. & \left.\theta_{1}, \mathbf{r}_{1}^{\prime} \theta_{1}^{\prime}, t\right) \\
= & N \int d \mathbf{r}_{2} d \theta_{2} d \mathbf{r}_{3} d \theta_{3} \ldots d \mathbf{r}_{N} d \theta_{N} \\
& \times \Phi^{*}\left(\mathbf{r}_{1}^{\prime} \theta_{1}^{\prime}, \mathbf{r}_{2} \theta_{2}, \ldots, \mathbf{r}_{N} \theta_{N}, t\right) \\
& \times H \Phi\left(\mathbf{r}_{1} \theta_{1}, \mathbf{r}_{2} \theta_{2}, \ldots, \mathbf{r}_{N} \theta_{N}, t\right) \\
& -N \int d \mathbf{r}_{2} d \theta_{2} d \mathbf{r}_{3} d \theta_{3} \ldots d \mathbf{r}_{N} d \theta_{N} \\
& \times\left[\Phi *\left(\mathbf{r}_{1} \theta_{1}, \mathbf{r}_{2} \theta_{2}, \ldots, \mathbf{r}_{N} \theta_{N}, t\right)\right. \\
& \left.\times H \Phi\left(\mathbf{r}_{1}^{\prime} \theta_{1}^{\prime}, \mathbf{r}_{2} \theta_{2}, \ldots, \mathbf{r}_{N} \theta_{N}, t\right)\right]^{*} .
\end{aligned}
$$

$\rho\left(\mathbf{r}_{1} \theta_{1}, \mathbf{r}_{1}^{\prime} \theta_{1}^{\prime}, t\right)$ may be expanded in the nonorthonormal AO basis set $\left\{\chi_{i}\right\}$ :

$$
\rho\left(\mathbf{r}_{1} \theta_{1}, \mathbf{r}_{1}^{\prime} \theta_{1}^{\prime}, t\right)=\sum_{i j}\left|\chi_{i}\left(\mathbf{r}_{1}\right)\right\rangle \rho_{i j}\left(\theta_{1}, \theta_{1}^{\prime}, t\right)\left\langle\chi_{j}\left(\mathbf{r}_{1}^{\prime}\right)\right|,
$$

where

$$
\rho_{i j}\left(\theta_{1}, \theta_{1}^{\prime}, t\right) \equiv \sum_{k}^{N} \sigma_{k}\left(\theta_{1}\right) c_{i k}^{\sigma_{k}}(t)\left[c_{j k}^{\sigma_{k}}(t)\right]^{*} \sigma_{k}^{*}\left(\theta_{1}^{\prime}\right)
$$

with $\sigma_{k}$ being the spin state of the $k$ th molecular spin-orbital $\psi_{k}$, see the Appendix. Integrating the right-hand-side (rhs) of Eq. (13), we obtain the EOM for the reduced singleelectron density matrix:

$$
i \hbar S \dot{\rho}^{\sigma} S=\left(h^{\sigma}+f\right) \rho^{\sigma} S-S \rho^{\sigma}\left(h^{\sigma}+f\right),
$$

where the reduced density matrix $\rho^{\sigma}$ for spin $\sigma$ is defined as

$$
\begin{aligned}
\rho_{i j}^{\sigma}(t) & \equiv\left\langle\sigma\left|\rho_{i j}\left(\theta, \theta^{\prime}, t\right)\right| \sigma\right\rangle \\
& =\sum_{l=\mathrm{occ}} c_{i l}^{\sigma}(t)\left[c_{j l}^{\sigma}(t)\right]^{*}
\end{aligned}
$$

with $l$ summing over the occupied spatial molecular orbitals, $h^{\sigma}$ is the Fock matrix whose elements are given as

$$
h_{n m}^{\sigma}(t)=t_{n m}+\sum_{i j, \sigma^{\prime}} \rho_{i j}^{\sigma^{\prime}}(t) V_{n m, i j}-\sum_{i j} \rho_{i j}^{\sigma}(t) V_{n i, j m},
$$

and $f$ characterizes the interaction between the $\pi$ electrons and the external field $\mathbf{E}(t)$ with its matrix elements being

$$
f_{n m}(t) \approx e \frac{z(n)+z(m)}{2} S_{n m} E(t) .
$$

Here we assume that the external electric field $\mathbf{E}(t)$ is polarized along the chain axis $z$. The detailed derivation of Eq. (16) is given in the Appendix. Since the systems that we are interested in are symmetric with respect to spin up and spin down, we neglect the spin index thereafter. We partition the density matrix $\rho(t)$ into two parts:

$$
\rho(t)=\rho^{(0)}+\delta \rho(t),
$$

where $\rho^{(0)}$ is the HF ground state reduced single-electron density matrix in the absence of external fields, and $\delta \rho(t)$ is the difference between $\rho(t)$ and $\rho^{(0)}$, i.e., the induced density matrix by the external field $\mathbf{E}(t)$. Similarly, the Fock matrix $h(t)$ is decomposed into the form,

$$
h(t)=h^{(0)}+\delta h(t)
$$

where $h^{(0)}$ is the Fock matrix when $\mathbf{E}(t)=0$ :

$$
h_{n m}^{(0)}=t_{n m}+\sum_{i j} \rho_{i j}^{(0)}\left(2 V_{n m, i j}-V_{n i, j m}\right)
$$

and the induced Fock matrix $\delta h$ is

$$
\delta h_{n m}(t)=\sum_{i j} \delta \rho_{i j}(t)\left(2 V_{n m, i j}-V_{n i, j m}\right) .
$$

With Eqs. (20) and (21), we can rewrite Eq. (16) as follows: 


$$
\begin{aligned}
i \hbar \delta \dot{\rho}= & {\left[S^{-1} h^{(0)} \delta \rho-\delta \rho h^{(0)} S^{-1}\right] } \\
& +\left[S^{-1} \delta h \rho^{(0)}-\rho^{(0)} \delta h S^{-1}\right] \\
& +\left[S^{-1} f \rho^{(0)}-\rho^{(0)} f S^{-1}\right] \\
& +\left[S^{-1} f \delta \rho-\delta \rho f S^{-1}\right] \\
& +\left[S^{-1} \delta h \delta \rho-\delta \rho \delta h S^{-1}\right] .
\end{aligned}
$$

For the first-order induced density matrix $\delta \rho^{(1)}$, its dynamics may be described by

$$
\begin{aligned}
i \hbar \delta \dot{\rho}^{(1)}= & {\left[S^{-1} h^{(0)} \delta \rho^{(1)}-\delta \rho^{(1)} h^{(0)} S^{-1}\right] } \\
& +\left[S^{-1} \delta h^{(1)} \rho^{(0)}-\rho^{(0)} \delta h^{(1)} S^{-1}\right] \\
& +\left[S^{-1} f \rho^{(0)}-\rho^{(0)} f S^{-1}\right] .
\end{aligned}
$$

More specifically,

$$
\begin{aligned}
i \hbar \delta \dot{\rho}_{i j}^{(1)}= & \sum_{k} \sum_{l}\left(S_{i k}^{-1} h_{k l}^{(0)} \delta \rho_{l j}^{(1)}-\delta \rho_{i k}^{(1)} h_{k l}^{(0)} S_{l j}^{-1}\right) \\
& +2 \sum_{k} \sum_{l} \sum_{m} \sum_{n}\left(S_{i k}^{-1} \delta \rho_{m n}^{(1)} V_{k l, m n} \rho_{l j}^{(0)}\right. \\
& \left.-\rho_{i k}^{(0)} \delta \rho_{m n}^{(1)} V_{k l, m n} S_{l j}^{-1}\right)-\sum_{k} \sum_{l} \sum_{m} \sum_{n}\left(S_{i k}^{-1}\right. \\
& \left.\times \delta \rho_{m n}^{(1)} V_{k m, n l} \rho_{l j}^{(0)}-\rho_{i k}^{(0)} \delta \rho_{m n}^{(1)} V_{k m, n l} S_{l j}^{-1}\right) \\
& +\sum_{k} \sum_{l}\left(S_{i k}^{-1} f_{k l} \rho_{l j}^{(0)}-\rho_{i k}^{(0)} f_{k l} S_{l j}^{-1}\right) .
\end{aligned}
$$

We integrate numerically Eq. (25) in the time domain, and solve it for the time evolution of the polarization vector $\mathbf{P}(t)$. Within the dipole approximation, $\mathbf{P}(t)$ may be expressed as

$$
\mathbf{P}(t)=\sum_{i j}-e\left\langle\chi_{i}|\hat{\mathbf{r}}| \chi_{j}\right\rangle \rho_{i j}(t) .
$$

Since we assume that the external electric field is polarized along the chain axis $z$, the first-order response $P_{z}^{(1)}$ is given by

$$
P_{z}^{(1)}(t) \approx \sum_{i j}-e \frac{z(i)+z(j)}{2} S_{i j} \delta \rho_{i j}^{(1)}(t) .
$$

To obtain the optical absorption spectrum, we then perform a Fourier transformation of $P_{z}^{(1)}(t)$,

$$
P_{z}^{(1)}(\omega)=\int_{-\infty}^{\infty} d t P_{z}^{(1)}(t) e^{-i \omega t} .
$$

The imaginary part $\alpha(\omega)$ of the complex linear polarizability is then determined readily via

$$
\alpha(\omega)=\operatorname{Im}\left[P_{z}^{(1)}(\omega) / E(\omega)\right],
$$

where $E(\omega)$ is the Fourier transform of $E(t)$.

\section{GENERALIZED LDM METHOD}

The key of the generalized LDM method is to reduce the dimension of the reduced single-electron density matrix, since the density matrix has a localized character or a "nearsightedness" not only for the ground state but also for lower excited states. This is achieved via the introduction of five critical lengths and related approximations. ${ }^{36,37}$

First, we set $\delta \rho_{i j}^{(1)}(t)$ to zero when $r_{i j}>l_{1}$. This approximation is based on the "near-sightedness" of $\delta \rho^{(1)}(t)$, and leads to the reduction of the number of unknown $\delta \rho_{i j}^{(1)}$ or the dimension of Eq. (26) from $N^{2}$ to $D \equiv\left(2 \alpha_{1}+1\right) N-\alpha_{1}\left(\alpha_{1}\right.$ +1 ), where $\alpha_{1}$ is the number of bonds within the distance $l_{1}$. Note $D$ scales linearly with $N$.

Second, $S_{i j}$ and $S_{i j}^{-1}$ are set to zero when $r_{i j}>l_{s 1}$ and $r_{i j}>l_{s 2}$, respectively. The overlap of two AOs decays rapidly with the increasing distance between them. Here $l_{s 1}$ is the critical length that characterizes the exponential decay of $S_{i j}$ with increasing $r_{i j}$. As it has been pointed out in Ref. 48, the off-diagonal element of $S_{i j}^{-1}$ diminishes exponentially for large $r_{i j}$ as well, and its decay is characterized by a slightly larger critical length $l_{s 2}$.

Third, $\rho_{i j}^{(0)}$ is set to zero when $r_{i j}>l_{0} . l_{0}$ is usually much longer than $l_{s 1}$ and $l_{s 2}$, i.e., $l_{0} \gg l_{s 1}$ and $l_{s 2}$ because $S_{i j}$ and $S_{i j}^{-1}$ decay rather rapidly with the increasing $r_{i j}$. According to Eq. (22), $h^{(0)}$ thus has approximately the same critical length $l_{0}$, i.e., $h_{i j}^{(0)}=0$ for $r_{i j}>l_{0}$.

For a fixed pair of $i$ and $j$, the second and third approximations result in the finite ranges of summations in Eq. (26) for $k, l, m$ and $n$ except the second term on the rhs of the equation. These finite ranges are determined by $l_{0}, l_{1}, l_{s 1}$ or $l_{s 2}$, and are approximately $2 \alpha_{1}, 2 \alpha_{0}, 2 \alpha_{s 1}$ or $2 \alpha_{s 2}$, respectively, where $\alpha_{0}, \alpha_{s 1}$ and $\alpha_{s 2}$ are the numbers of bonds within $l_{0}, l_{s 1}$ and $l_{s 2}$, respectively. However, the total number of summations in the second term on the rhs of Eq. (26)

$$
2 \sum_{k} \sum_{l} \sum_{m} \sum_{n}\left(S_{i k}^{-1} \delta \rho_{m n}^{(1)} V_{k l, m n} \rho_{l j}^{(0)}-\rho_{i k}^{(0)} \delta \rho_{m n}^{(1)} V_{k l, m n} S_{l j}^{-1}\right)
$$

is proportional to $N$, since the number of summations over $m$ and $n$ is of $O(N)$. To achieve the linear scaling of the computational time, the number of summations over $m$ and $n$ must be limited to a fixed value which does not vary with $N$. There are two types of cancellation in Eq. (31). (i) The sum of $S_{i k}^{-1} V_{k l, m n} \rho_{l j}^{(0)}$ and $-\rho_{i k}^{(0)} V_{k l, m n} S_{l j}^{-1}$ cancels much of their values; (ii) since $\Sigma_{m n} \delta \rho_{n m}^{(1)}=0$, i.e., the charge conservation, the summation over $m$ and $n$ leads to further cancellation. Therefore, we may limit $m(n)$ between $m_{0}\left(n_{0}\right)$ and $m_{1}$ $\left(n_{1}\right)$, where $m_{0}=n_{0}=\max \left[1, \min \left(i-\alpha_{c}-\alpha_{s 2}-\frac{1}{2} \alpha_{s 1}, j-\alpha_{c}\right.\right.$ $\left.\left.-\alpha_{s 2}-\frac{1}{2} \alpha_{s 1}\right)\right]$ and $m_{1}=n_{1}=\min \left[N, \max \left(i+\alpha_{c}+\alpha_{s 2}+\frac{1}{2} \alpha_{s 1}, j\right.\right.$ $\left.\left.+\alpha_{c}+\alpha_{s 2}+\frac{1}{2} \alpha_{s 1}\right)\right] . \alpha_{c}$ is the number of bonds within a distance $l_{c}$, and $l_{c}$ is the critical length that limits the sumation ranges of $m$ and $n$ beyond which cancellations (i) and (ii) render further summation negligible. This is our fourth approximation.

The first, third and fourth approximations are exactly the same as those in Refs. 36 and 37. The second approximation is due to the use of the nonorthonormal basis set and the consequent introduction of the overlap matrix $S$. With these approximations, Eq. (26) becomes 


$$
\begin{aligned}
& i\left(\hbar \frac{d}{d t}+\gamma\right) \delta \rho_{i j}^{(1)}=\sum_{k}^{|i-k| \leqslant \alpha_{s 2},|k-l| \leqslant \alpha_{0},|l-j| \leqslant \alpha_{1}} \sum_{l}^{-1} S_{i k}^{-1} h_{k l}^{(0)} \delta \rho_{l j}^{(1)}-\sum_{k}^{|i-k| \leqslant \alpha_{1},|k-l| \leqslant \alpha_{0},|l-j| \leqslant \alpha_{s 2}} \sum_{l} \delta \rho_{i k}^{(1)} h_{k l}^{(0)} S_{l j}^{-1}
\end{aligned}
$$

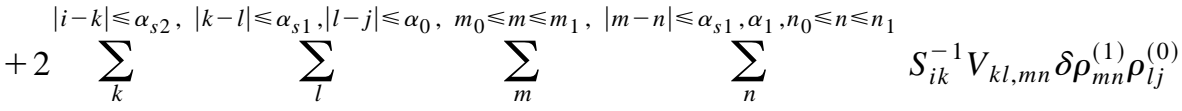

$$
\begin{aligned}
& -2 \sum_{k}^{|i-k| \leqslant \alpha_{0},|k-l| \leqslant \alpha_{s 1},|l-j| \leqslant \alpha_{s 2},} \sum_{l}^{m_{0} \leqslant m \leqslant m_{1},|m-n| \leqslant \alpha_{s 1}, \alpha_{1}, n_{0} \leqslant n \leqslant n_{1}} \sum_{n}^{(0)} \rho_{i k}^{(0)} V_{k l, m n} \delta \boldsymbol{\rho}_{m n}^{(1)} S_{l j}^{-1} \\
& |i-k| \leqslant \alpha_{s 2},|k-m| \leqslant \alpha_{s 1},|m-n| \leqslant \alpha_{1},|n-l| \leqslant \alpha_{s 1},|l-j| \leqslant \alpha_{0} \\
& -\sum_{k} \sum_{m} \sum_{n} \sum_{l} S_{i k}^{-1} V_{k m, n l} \delta \rho_{m n}^{(1)} \rho_{l j}^{(0)} \\
& |i-k| \leqslant \alpha_{0},|k-m| \leqslant \alpha_{s 1},|m-n| \leqslant \alpha_{1},|n-l| \leqslant \alpha_{s 1},|l-j| \leqslant \alpha_{s 2} \\
& +\sum_{k} \quad \sum_{m} \quad \sum_{n} \quad \sum_{l} \rho_{i k}^{(0)} V_{k m, n l} \delta \rho_{m n}^{(1)} S_{l j}^{-1} \\
& |i-k| \leqslant \alpha_{s 2},|k-l| \leqslant \alpha_{s 1},|l-j| \leqslant \alpha_{0} \quad|i-k| \leqslant \alpha_{0},|k-l| \leqslant \alpha_{s 1},|l-j| \leqslant \alpha_{s 2} \\
& +\sum_{k} \quad \sum_{l} S_{i k}^{-1} f_{k l} \rho_{l j}^{(0)}-\sum_{k} \sum_{l} \rho_{i k}^{(0)} f_{k l} S_{l j}^{-1} \text {. }
\end{aligned}
$$

In Eq. (32) the summation over $k, l, m$, and $n$ are restricted to the finite ranges which do not depend on the value of $N$. Since the number of $\delta \rho_{i j}^{(1)}$ is proportional to $N$, the total number of steps required to integrate Eq. (32) scales linearly with $N$. Therefore, we expect that the computational time is proportional to $N$.

We include explicitly the phenomenological dephasing $\gamma$ in Eq. (32). In the calculation, we use the fourth-order Runge-Kutta method ${ }^{49}$ for solving Eq. (32). We have used the external field,

$$
\mathcal{E}(t)=\frac{1}{\sqrt{\pi} \bar{t}} e^{-(t / \bar{t})^{2}},
$$

where $\bar{t}=0.1$ fs and perform the time integration for the time duration between -0.5 and $220 \mathrm{fs}$ with the time step $0.025 \mathrm{fs}$ to calculate the absorption spectra. The phenomenological dephasing $\gamma$ is set to $25 \mathrm{meV}$.

\section{EFFECTIVE HAMILTONIAN FOR $\pi$ ELECTRONS}

The PPP model is widely used to describe the $\pi$ electron system of planar conjugated molecules. ${ }^{26}$ The orthogonalized AOs are employed in the PPP model. Chen and Mulamel ${ }^{50}$ have developed the constrained density matrix variation $(\mathrm{CDMV})^{51}$ approach to determine the effective Hamiltonians of reduced electronic systems, and applied it to PA to obtain the PPP-like Hamiltonian for the $\pi$ electrons. The natural atomic orbitals (NAOs) were used as the basis set. In this work the nonorthonormal $\pi$ AOs are used as the basis set. A new effective Hamiltonian with the nonorthonormal basis set is thus required. We describe here briefly the CDMV approach that we use to determine the effective Hamiltonian with the nonorthonormal $\pi$ AO basis set. becomes

When there is no external field, i.e., $\mathbf{E}(t)=0$, Eq. (16)

$$
h^{(0)} \rho^{(0)} S-S \rho^{(0)} h^{(0)}=0 .
$$

This equation can be recast in the form

$$
d_{m n}=\sum_{i} t_{m i} P_{L}(i, n)-\sum_{i} P_{R}(m, i) t_{i n}+R_{m n}=0,
$$

where

$$
\begin{aligned}
R_{m n}= & 2 \sum_{i k l} V_{m i, k l} \rho_{k l}^{(0)} P_{L}(i, n)-2 \sum_{i k l} P_{R}(m, i) V_{i n, k l} \rho_{k l}^{(0)} \\
& -\sum_{i k l} \rho_{k l}^{(0)} V_{m k, l i} P_{L}(i, n)+\sum_{i k l} P_{R}(m, i) \rho_{k l}^{(0)} V_{i k, l n},
\end{aligned}
$$

$$
P_{L}(i, j)=\sum_{k} \rho_{i k}^{(0)} S_{k j},
$$

$$
P_{R}(i, j)=\sum_{k} S_{i k} \rho_{k j}^{(0)}
$$

As in Ref. 50, a function $\mathcal{G}$ is constructed:

$$
\mathcal{G}=\sum_{m n} d_{m n}^{2}+\mathcal{F},
$$

where $\mathcal{F}$ represents the variational constraints. ${ }^{50} t_{m n}$ is determined by minimizing the value of $\mathcal{G}$. Upon minimizing $\mathcal{G}$ with respect to $t_{i j}$, we have

$$
\frac{\partial \mathcal{G}}{\partial t_{i j}}=2 \sum_{m n} d_{m n} \frac{\partial d_{m n}}{\partial t_{i j}}+\frac{\partial \mathcal{F}}{\partial t_{i j}}=0 .
$$

The effective Hamiltonian is determined by solving Eq. (40) for $t_{i j}$. With $\mathcal{F}=0$, Eq. (40) becomes 
TABLE I. $t_{i j}$ of the effective Hamiltonian for $\pi$ electrons with 40 carbon atoms (in $\mathrm{eV}$ ). (The system is symmetric. Thus $t_{i j}=t_{\bar{i}, \bar{j}}$ for $\bar{j}=N+1-j$ and $\bar{i}=N+1-i$ ).

\begin{tabular}{crrrrrrrrrr}
\hline \hline$i$ & 2 & 3 & 4 & 5 & 6 & 7 & 8 & 9 & 10 & \\
\hline$t_{i, i-1}$ & -1.986 & -1.371 & -2.198 & -1.496 & -2.295 & -1.567 & -2.363 & -1.619 & -2.419 & \\
$t_{i, i}$ & 0.030 & 0.069 & 0.085 & 0.107 & 0.116 & 0.131 & 0.137 & 0.148 & 0.152 & \\
& & & & & & & & & & \\
$i$ & 11 & 12 & 13 & 14 & 15 & 16 & 17 & 18 & 19 & 20 \\
\hline$t_{i, i-1}$ & -1.660 & -2.466 & -1.694 & -2.508 & -1.722 & -2.539 & -1.740 & -2.560 & -1.753 & -2.573 \\
$t_{i, i}$ & 0.160 & 0.163 & 0.170 & 0.172 & 0.176 & 0.177 & 0.181 & 0.181 & 0.183 & 0.183 \\
\hline \hline
\end{tabular}

$$
\begin{aligned}
\sum_{m k} t_{i k} P_{L}(k, m) P_{L}(j, m)+\sum_{m k} t_{k j} P_{R}(m, k) P_{R}(m, i) \\
\quad-\sum_{m k} t_{k m} P_{R}(i, k) P_{L}(j, m)-\sum_{m k} t_{m k} P_{L}(k, j) P_{R}(m, i) \\
+\sum_{m} R_{i m} P_{L}(j, m)-\sum_{m} R_{m j} P_{R}(m, i)=0 .
\end{aligned}
$$

We keep only those $t_{i j}$ that represent the local $\pi$ atomic energies and one-electron integrals across the nearest neighbors. Thus, we set

$$
t_{i j}=0
$$

for $j \neq i \pm 1$ or $j \neq i$, and solve Eq. (41) for $t_{i i}$ and $t_{i, i \pm 1} \cdot \rho^{(0)}$ is the input, and may be obtained from the ab initio calculations. The effective Coulomb interaction among the $\pi$ electrons may be approximated by Eq. (9). $a_{0}$ is set to $1.29 \AA$. $U$ is to be chosen so that the calculated optical gap fits the experimental value. In the calculation $t_{11}$ is set to zero since only the relative energies are of physical interest.

We determine first the effective Hamiltonian with the $\pi$ AOs as the basis set. A PA oligomer with 40 carbon atoms is chosen. It is found that $U=1.81 \mathrm{eV}$ results in an optical gap of $2.23 \mathrm{eV}$ for $N=40$ and leads to $\sim 2.0 \mathrm{eV}$ for PA $(N$ $\rightarrow \infty)$. Resulting values of $t_{i j}$ are listed in Table I. $t_{i i}^{*}$ is the bare AO energy of $i^{50}$ which may be written as follows:

$$
t_{i, i}^{*}=t_{i, i}+\sum_{k}\left(V_{i i, k k}-V_{11, k k}\right) .
$$

The resulting Hamiltonian is used to calculate the HF ground state reduced single-electron density matrix which is then compared with the $a b$ initio HF ground state reduced singleelectron density matrix (see Table II). Since the oligomer is centro-symmetric, we list only data for $i=1$ to 20 . We calculate the effective Hamiltonian with even number $N, N$ $=2 n=8 \rightarrow 48$, and find that $t_{i j}$ converged at $N \sim 32$. The effective Hamiltonians for a larger system $(N>40)$ may thus be determined from that of the $N=40$. To construct the effective Hamiltonians for longer oligomers, we follow the strategy below:

the values of the first $20 t_{i, i \pm 1}$ and $t_{i i}^{*}$ from each end of the oligomer are given in Table I;

the rest of the bare orbital energy, one-electron inte- grals for the double and single bonds are taken as $0.183,-2.573$ and $-1.753 \mathrm{eV}$, respectively.

The resulting Hamiltonians are used to calculate the optical spectra of longer oligomers. The details of the calculation that determines the effective Hamiltonian will appear in a separate publication. ${ }^{52}$

\section{RESULTS}

The GAUSSIAN 94 software package is employed to calculate the overlap matrix $S$ and the $a b$ initio HF ground state reduced single-electron density matrix $\rho^{(0)}$. Geometry optimization is performed at the HF level. All the double or single bond lengths are kept the same, and the bond angles between the double and single bonds are $124.02^{\circ}$. For $N$ $=40$, we find that the double and single bond lengths are 1.324 and $1.478 \AA$, respectively. $S_{i j}$ or $S_{i j}^{-1}$ decreases quickly to zero with the increasing $r_{i j}$. For instance,

(i) $S_{10,11}=0.184$ and $S_{10,11}^{-1}=-0.203$. (ii) $S_{11,12}=0.245$ and $S_{11,12}^{-1}=-0.270$. (iii) $S_{10,12}=0.023$ and $S_{10,12}^{-1}=0.025$. Thus, relatively short $l_{s 1}$ and $l_{s 2}(\sim 10 \AA)$ may be used to truncate the $S$ and $S^{-1}$, respectively. In Table II, we list the diagonal and nearest-neighbor off-diagonal density matrix elements $\rho_{i i}^{(0)}$ and $\rho_{i, i+1}^{(0)}$ for $N=40$. Note that the diagonal elements $\rho_{i i}^{(0)}$ are equal to 0.5 , and are approximately 0.398 when $i$ is not at or near either end of the oligomer. The electron density $n_{i}$ at the $i$ th orbital may be calculated through the following formula:

$$
n_{i}=\sum_{j} \rho_{i j}^{(0)} S_{j i}
$$

The resulting values of $n_{i}$ 's are 0.5 except that $i=1$, and are listed in Table II.

The HF method is a self-consistent-field (SCF) method whose solution requires an initial guess of the density matrix. To construct the initial ground state density matrix for $N$ $>40$, the following procedure is employed. First, we calculate the reduced single electron density matrix of a PA oligomer with 40 carbon atoms using the GAUSSIAN 94 program. It is shown via a two-dimensional contour plot in Fig 1. Note that the density matrix is band diagonal, and outside the diagonal band the matrix elements are almost zero. This is the so-called "near-sightedness" of $\rho^{(0)}$, and the width of the band is $\sim 2 \alpha_{0}$. We note further that the middle part of the diagonal band is quite homogeneous with a period of 4 , see 
TABLE II. The diagonal and the nearest neighbor off-diagonal elements of the ground state reduced single-electron density matrix $\rho_{i j}^{(0)},{ }^{\mathrm{a}}$ and the charge density $n_{i} \cdot{ }^{\mathrm{b}}$

\begin{tabular}{|c|c|c|c|c|c|c|c|c|c|c|}
\hline$i$ & 1 & 2 & 3 & 4 & 5 & 6 & 7 & 8 & 9 & 10 \\
\hline$\rho_{i i}^{(0)}$ & $\begin{array}{c}0.416 \\
(-0.005)\end{array}$ & $\begin{array}{c}0.389 \\
(0.003)\end{array}$ & $\begin{array}{r}0.399 \\
(-0.003)\end{array}$ & $\begin{array}{c}0.397 \\
(0.004)\end{array}$ & $\begin{array}{c}0.398 \\
(-0.001)\end{array}$ & $\begin{array}{c}0.397 \\
(0.003)\end{array}$ & $\begin{array}{c}0.398 \\
(-0.001)\end{array}$ & $\begin{array}{c}0.398 \\
(0.002)\end{array}$ & $\begin{array}{c}0.398 \\
(-0.000)\end{array}$ & $\begin{array}{c}0.398 \\
(0.002)\end{array}$ \\
\hline$\rho_{i i+1}^{(0)}$ & $\begin{array}{c}0.385 \\
(0.004)\end{array}$ & $\begin{array}{c}0.072 \\
(-0.011)\end{array}$ & $\begin{array}{c}0.368 \\
(0.008)\end{array}$ & $\begin{array}{c}0.078 \\
(-0.015)\end{array}$ & $\begin{array}{c}0.366 \\
(0.010)\end{array}$ & $\begin{array}{c}0.080 \\
(-0.017)\end{array}$ & $\begin{array}{c}0.366 \\
(0.011)\end{array}$ & $\begin{array}{c}0.079 \\
(-0.019)\end{array}$ & $\begin{array}{c}0.366 \\
(0.012)\end{array}$ & $\begin{array}{r}0.080 \\
(-0.020)\end{array}$ \\
\hline$n_{i}$ & $\begin{array}{c}0.505 \\
(-0.004)\end{array}$ & $\begin{array}{c}0.496 \\
(0.002)\end{array}$ & $\begin{array}{c}0.500 \\
(-0.003)\end{array}$ & $\begin{array}{c}0.499 \\
(0.003)\end{array}$ & $\begin{array}{c}0.500 \\
(-0.002)\end{array}$ & $\begin{array}{c}0.500 \\
(0.002)\end{array}$ & $\begin{array}{c}0.500 \\
(-0.001)\end{array}$ & $\begin{array}{c}0.500 \\
(0.002)\end{array}$ & $\begin{array}{c}0.500 \\
(-0.001)\end{array}$ & $\begin{array}{c}0.500 \\
(0.001)\end{array}$ \\
\hline$i$ & 11 & 12 & 13 & 14 & 15 & 16 & 17 & 18 & 19 & 20 \\
\hline$\rho_{i i}^{(0)}$ & $\begin{array}{c}0.398 \\
(0.000)\end{array}$ & $\begin{array}{c}0.398 \\
(0.002)\end{array}$ & $\begin{array}{c}0.398 \\
(0.000)\end{array}$ & $\begin{array}{c}0.398 \\
(0.001)\end{array}$ & $\begin{array}{c}0.398 \\
(0.001)\end{array}$ & $\begin{array}{c}0.398 \\
(0.001)\end{array}$ & $\begin{array}{c}0.398 \\
(0.001)\end{array}$ & $\begin{array}{c}0.398 \\
(0.001)\end{array}$ & $\begin{array}{c}0.398 \\
(0.001)\end{array}$ & $\begin{array}{c}0.398 \\
(0.001)\end{array}$ \\
\hline$\rho_{i i+1}^{(0)}$ & $\begin{array}{c}0.365 \\
(0.012)\end{array}$ & $\begin{array}{c}0.080 \\
(-0.020)\end{array}$ & $\begin{array}{c}0.366 \\
(0.013)\end{array}$ & $\begin{array}{c}0.080 \\
(-0.021)\end{array}$ & $\begin{array}{c}0.366 \\
(0.013)\end{array}$ & $\begin{array}{c}0.080 \\
(-0.021)\end{array}$ & $\begin{array}{c}0.366 \\
(0.013)\end{array}$ & $\begin{array}{c}0.080 \\
(-0.021)\end{array}$ & $\begin{array}{c}0.366 \\
(0.013)\end{array}$ & $\begin{array}{r}0.080 \\
(-0.021)\end{array}$ \\
\hline$n_{i}$ & $\begin{array}{c}0.500 \\
(-0.001)\end{array}$ & $\begin{array}{c}0.500 \\
(0.001)\end{array}$ & $\begin{array}{c}0.500 \\
(-0.000)\end{array}$ & $\begin{array}{c}0.500 \\
(0.001)\end{array}$ & $\begin{array}{c}0.500 \\
(-0.000)\end{array}$ & $\begin{array}{c}0.500 \\
(0.000)\end{array}$ & $\begin{array}{c}0.500 \\
(-0.000)\end{array}$ & $\begin{array}{c}0.500 \\
(0.000)\end{array}$ & $\begin{array}{c}0.500 \\
(0.000)\end{array}$ & $\begin{array}{c}0.500 \\
(0.000)\end{array}$ \\
\hline
\end{tabular}

${ }^{\mathrm{a}} \rho_{i j}^{(0)}$ is the reduced density matrix elements given by ab initio calculation using GAUSSIAN 94.

${ }^{\mathrm{b}} n_{i}$ is the charge on each site: $n_{i}=\Sigma_{j} \rho_{i j}^{(0)} S_{i j}$. Data in the parentheses below each value is the difference between the $a b$ initio result and its counterpart calculated from the effective Hamiltonian.

Fig. 2. The period of 4 reflects the fact that PA has the repeating double and single bond structure. We thus elongate the diagonal band by repeatedly inserting the period until the density matrix reaches the desired size, see Fig. 2. The resulting density matrix is used as the initial guess for the ground state density matrix of the large system $(N>40)$.

The inset of Fig. 3 shows the time evolution of polarization $P_{z}^{(1)}(t)$ for $N=120$ for $\alpha_{1}=40, \alpha_{0}=\alpha_{c}=24, \alpha_{s 1}=4$, and $\alpha_{s 2}=8 . P_{z}^{(1)}(t)$ oscillates with time and its oscillation amplitude decays as $e^{-\gamma t}$. From the Fourier transform of $P_{z}^{(1)}(t)$, we obtain the absorption spectrum [see Eqs. (29) and (30)]. Figure 3 shows the absorption spectrum for $N$ $=120$ with two sets of $\alpha_{0}, \alpha_{1}, \alpha_{c}, \alpha_{s 1}$, and $\alpha_{s 2} . \alpha_{0}=\alpha_{c}$ $=24, \alpha_{s 1}=4$ and $\alpha_{s 2}=8$ are employed. The diamonds are for $\alpha_{1}=30$, and the triangles are for $\alpha_{1}=40$. Clearly, the two sets of data agree well with each other. Thus, the critical length $l_{1}$ of $\delta \rho^{(1)}$ covers about 30 double or single bonds, i.e., $\alpha_{1}=30$ results in an accurate absorption spectrum up to a frequency of $2.3 \mathrm{eV}$. The absorption peak in Fig. 3 corresponds to the excited state $1 B_{u}$. For higher frequency range, we find that larger $\alpha_{1}$ is required to produce an accurate absorption spectrum. This implies that the density matrices of the higher excited states have longer $l_{1}$.

To investigate the roles of $\alpha_{0}$ and $\alpha_{c}$ on the accuracy of the calculation, we compare the absorption spectra for three

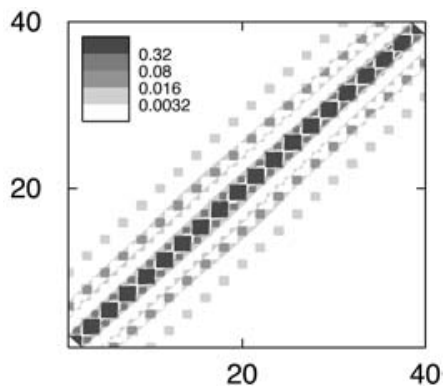

FIG. 1. The reduced ground state density matrix for $N=40$. different sets of $\alpha_{0}$ and $\alpha_{c}$ with $\alpha_{1}, \alpha_{s 1}$ and $\alpha_{s 2}$ being fixed at 40,4 , and 8 , respectively. The resulting absorption spectra are shown in Fig. 4. The solid line is for $\alpha_{0}=30$ and $\alpha_{c}$ $=24$, the diamonds for $\alpha_{0}=\alpha_{c}=24$, and the crosses for $\alpha_{0}$ $=24$ and $\alpha_{c}=30$. Obviously, the three sets of data for the absorption spectrum are virtually the same. This implies strongly that $\alpha_{0}=\alpha_{c}=24$ is sufficient to yield an accurate absorption spectrum. Moreover, it verifies that our fourth approximation in Sec. IV is very reliable.

To demonstrate that the computational time of the generalized LDM method scales linearly with the system size $N$, we calculate the linear response to the external field $\mathbf{E}(\mathbf{t})$ for $N=40,80,120,160,200,300,380$ and 500. $\alpha_{0}=\alpha_{c}=\alpha_{1}$ $=20, \alpha_{s 1}=2$ and $\alpha_{s 2}=4$ are employed. The CPU time for each calculation is measured, and the results are plotted with a dashed line in Fig. 5. The CPU time spent in the HF ground state has been subtracted from the total CPU time. So the resulting CPU time in Fig. 5 is for the excited states or the optical response only. Clearly, the linear scaling of the computational time versus the system size is achieved for the

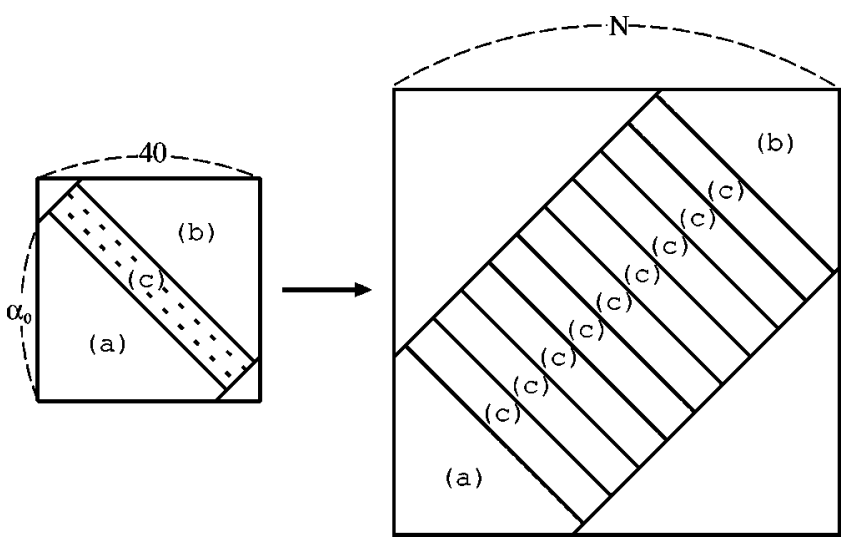

FIG. 2. Constructing density matrix for a larger system from the density matrix for $N=40$. During the construction (a) and (b) are kept unchanged; (c) is repeated until the density matrix reaches the desirable size. 


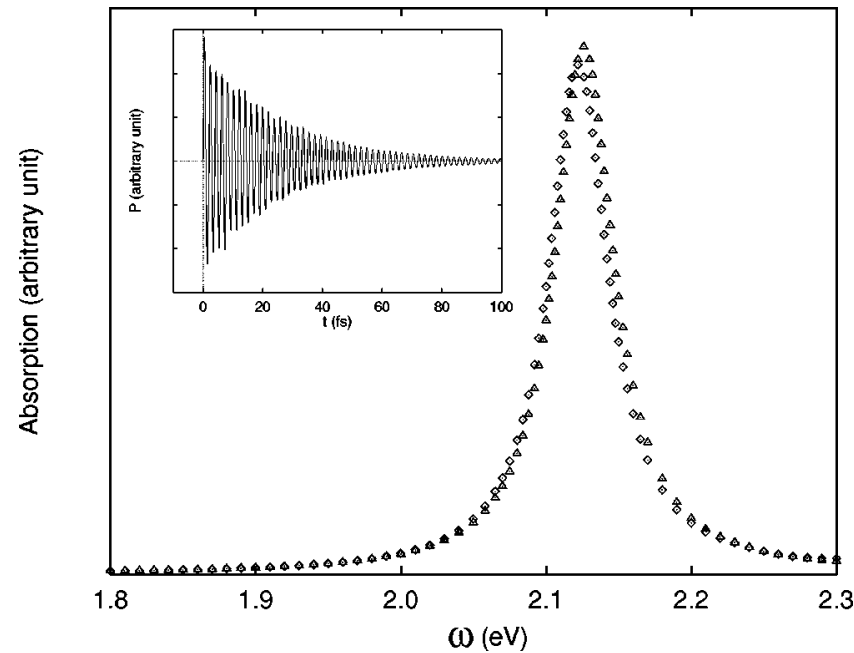

FIG. 3. Absorption spectra for $N=120$ with different $\alpha_{1} . \alpha_{0}=\alpha_{c}=24$, $\alpha_{s_{1}}=4$ and $\alpha_{s_{2}}=8$. The diamonds are for $\alpha_{1}=30$ and the triangles are for $\alpha_{1}=40$. The inset shows the time evolution of polarization $P_{z}^{(1)}$ for $\alpha_{1}$ $=40$. The phenomenological dephasing constant $\gamma=25 \mathrm{meV}$.

excited state properties. For the comparison the CPU time for the full TDHF is shown by the dotted line which has an $O\left(N^{4}\right)$ scaling. We can clearly see the drastic reduction of the CPU time for the LDM method as compared to the full TDHF method. Note that the LDM method is also faster even for the small systems. This is always true for onedimensional systems where the indices of atomic orbitals may be assigned in a simple increasing order along the system axis. However, for two- or three- dimensional systems, this does not usually hold which may lead to additional computational cost for the LDM method, and a CPU time crossover between the LDM method and the full TDHF may occur.

The computational time dependence on the values of $\alpha_{0}$, $\alpha_{1}$ and $\alpha_{c}$ is studied as well. In Figs. 6, 7, and 8 we plot the CPU time versus $\alpha_{1}, \alpha_{c}$ and $\alpha_{0}$, respectively. The diamonds

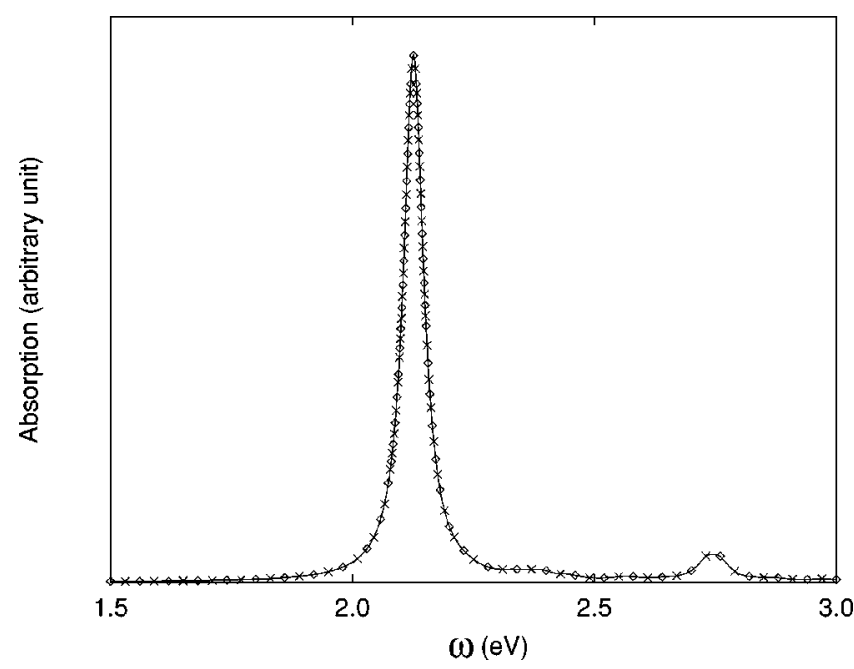

FIG. 4. Absorption spectra for $N=120$ with different $\alpha_{0}$ and $\alpha_{c} . \alpha_{1}=40$, $\alpha_{s_{1}}=4$ and $\alpha_{s_{2}}=8$. The diamonds are for $\alpha_{0}=\alpha_{c}=24$. The crosses are for $\alpha_{0}=24, \alpha_{c}=30$. The solid line is for $\alpha_{0}=30, \alpha_{c}=24$. The phenomenological dephasing constant $\gamma=25 \mathrm{meV}$.

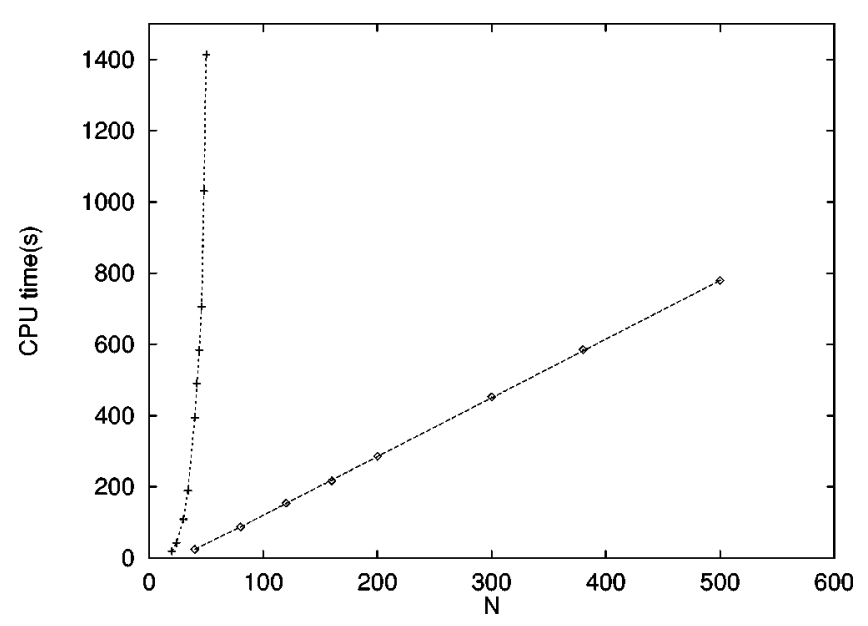

FIG. 5. CPU time of LDM on an SGI Indigo2 R10000 workstation for $N$ $=40,80,120,160,200,300,380$ and 500 (the dashed line). $\alpha_{0}=\alpha_{c}=\alpha_{1}$ $=20, \alpha_{s_{1}}=2$ and $\alpha_{s_{2}}=4$. The full TDHF calculation is shown by the dotted line. Each calculation is performed during the time interval between -0.5 and 0.25 fs with the time step 0.025 fs. $\gamma=25 \mathrm{meV}$.

are the resulting CPU times. The dashed lines are the least fits to the data assuming that the CPU time depends linearly on $\alpha_{1}, \alpha_{c}$ and $\alpha_{0}$. The computational time scales linearly with $\alpha_{1}$ and $\alpha_{c}$ with the ranges of values studied. For $\alpha_{0}$, the $O\left(\alpha_{0}\right)$ scaling of the CPU time holds approximately.

\section{DISCUSSION}

The fourth approximation in Sec. IV may not seem to be straightforward or intuitive. In fact, it is an excellent approximation. The justification of the approximation comes mainly from the cancellation (ii) which is caused by the charge conservation (i.e., $\Sigma_{n} \delta \rho_{n n}^{(1)}=0$ ). The different values of $\alpha_{c}$ result in virtually the same absorption spectra for $N=120$, see Fig. 4. For the frequency from 1.5 to $10 \mathrm{eV}$, the results for $\alpha_{c}$ $=24$ and 30 differ from each other by less than $0.1 \%$. This fact illustrates convincingly the validity of our fourth ap-

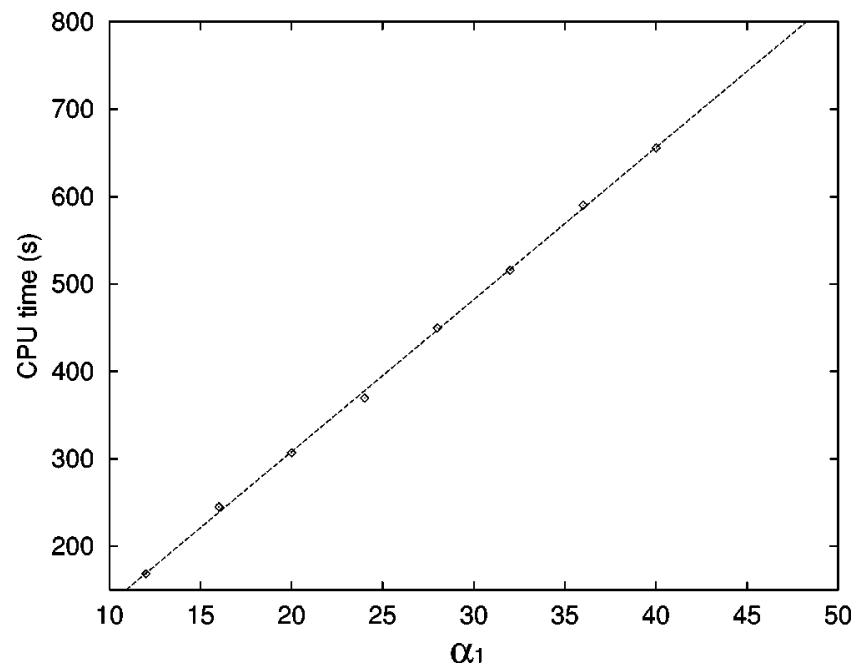

FIG. 6. CPU time on an SGI Origin 200 workstation for different $\alpha_{1} . N$ 120. $\gamma=25 \mathrm{meV} . \alpha_{0}=\alpha_{c}=24, \alpha_{s_{1}}=4$ and $\alpha_{s_{2}}=8$. Each calculation is performed during the time interval between -0.5 and $0.25 \mathrm{fs}$ with time step $0.025 \mathrm{fs}$. 


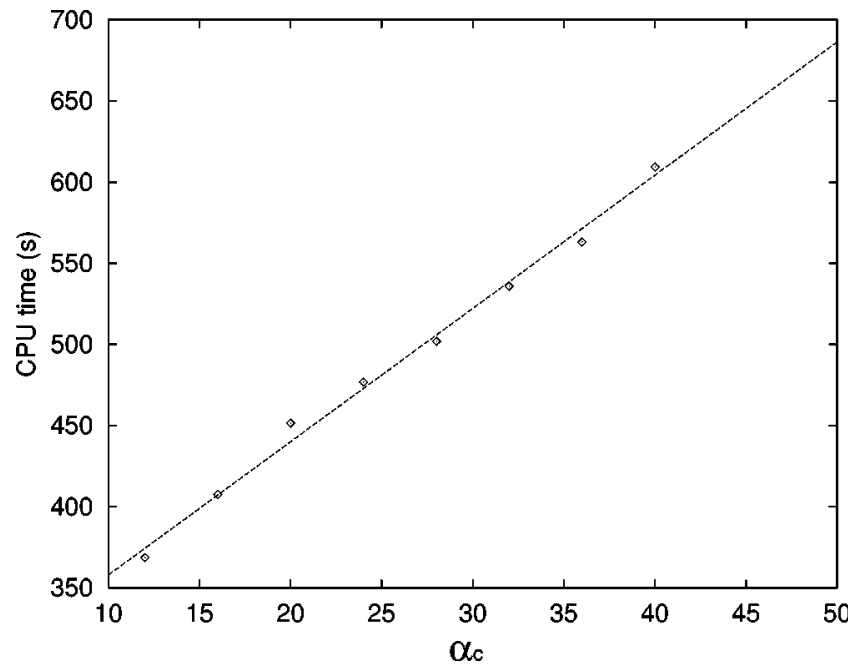

FIG. 7. CPU time on an SGI Origin 200 workstation for different $\alpha_{c} . N$ $=120 . \gamma=25 \mathrm{meV} . \alpha_{0}=24, \alpha_{1}=30, \alpha_{s_{1}}=4$ and $\alpha_{s_{2}}=8$. Each calculation is performed during the time interval between -0.5 and $0.25 \mathrm{fs}$ with time step 0.025 fs.

proximation or the introduction of the critical length $l_{c}$. When the cancellation is strong, $l_{c} \approx l_{0}$; when the cancellation is weak, $l_{c} \gg l_{0}$ is expected. The fast multiple method (FMM) has been used to calculate the summation of Coulomb interaction, ${ }^{19,53,54}$ and its computational time scales linearly with the system size $N .^{19,54}$ It may be one of the alternative ways to calculate Eq. (31). The values of the critical lengths $l_{0}$ and $l_{1}$ (or, $\alpha_{0}$ and $\alpha_{1}$ ) are determined empirically. For instance, we set $\alpha_{1}=30$ and 40 and calculate the absorption spectra, respectively. We find that the two resulting absorption spectra differ little, and thus, conclude that $\alpha_{1}=40$ is a good critical length for the first-order induced density matrix, which is employed in the subsequent calculations. Although the band diagonal form is utilized to achieve the $O(N)$ scaling in Ref. 36, it is not necessary when Eq. (26) is solved in the time domain. Since the critical lengths are roughly independent of the dimensionality of the system, the product of truncated matrices requires only the multiplication of the matrix elements within the critical lengths. This would lead to the $O(N)$ scaling of computation time even for twoand three-dimensional systems, although a larger overhead of computational effort may be required. Therefore, the method may be extended to two- and three-dimensional systems, and a variety of physical, chemical, or biological systems may be investigated with this method. To probe more excited states, we may generalize our current method to calculate the higher order responses. For the first order response, only the first term on the rhs of Eq. (24) contributes. For the higher order responses, the second and third terms on the rhs contribute as well. With the truncation of density matrix and Fock matrix, the computational time spent in evaluating the second and third terms is proportional to $N$. The computation for the higher order responses is thus of $O(N)$ scaling as well. In our calculation, the HF ground state is obtained first. This part of the calculation scales as $O\left(N^{3}\right)$. However, compared with the total time, its computational time is trivial for $N=40$ to 500 . The HF ground state reduced

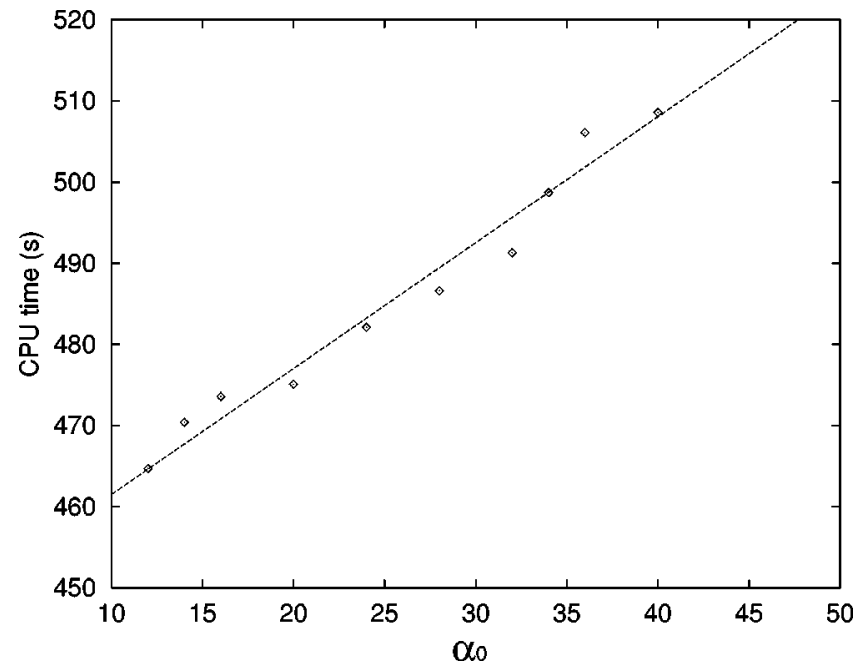

FIG. 8. CPU time on an SGI Origin 200 workstation for different $\alpha_{0}$. $N$ $=120 . \gamma=25 \mathrm{meV} . \alpha_{c}=24, \alpha_{1}=30, \alpha_{s_{1}}=4$ and $\alpha_{s_{2}}=8$. Each calculation is performed during the time interval between -0.5 and $0.25 \mathrm{fs}$ with time step $0.025 \mathrm{fs}$.

density matrix may be calculated via the iterative usage of Eq. (34) starting with a reasonable guess for the reduced density matrix. ${ }^{55}$ Combining our method for the excited states with the linear scaling algorithms for the ground state ${ }^{55,1-23}$ would lead to a linear scaling of the total computational time. In our calculation, we observed that for the frequency below $3.0 \mathrm{eV}$ the first-order induced density matrix is localized within a critical length of $42 \AA$. For higher frequency modes, the induced density matrices have larger critical lengths, ${ }^{35}$ and thus, more computational time is required. For extremely high energy modes, the induced density matrices may spread over the entire molecule, ${ }^{35}$ and therefore, the full TDHF calculation is required.

The overlap matrix $S$ is introduced because the nonorthonormal basis set is employed. This leads to an increase of the computational time. However, the increase is limited. Since the overlap matrix element $S_{i j}$ diminishes rapidly as the distance between $i$ and $j$ increases, only the overlaps among few nearby atoms are considered. The inclusion of the differential overlap integrals together with the usage of the nonorthonormal basis set makes it possible to implement the LDM at the $a b$ initio and semiempirical calculation levels. Since the linear scaling calculation nature of the LDM is not altered by the usage of the nonorthonormal basis set and the inclusion of complete differential overlap integrals, it is practical to achieve the linear scaling calculation for the excited state properties at the $a b$ initio and semiempirical levels. No further approximation is made for the Hamiltonian. The approximations are based solely on the feature of the reduced density matrix. This fact ensures the wide applicability of the new method.

The one-electron integrals of the effective Hamiltonian obtained in this work are similar to that of Ref. 50 while the two-electron integrals are much smaller. This is caused by the inclusion of the overlap matrix $S$ and the differential overlap integrals. To improve the accuracy of the effective Hamiltonians, the one-electron integrals other than those of 
the nearest neighbors should be included, ${ }^{56}$ and moreover the CDMV approach should be extended beyond the HF level to include the electron-electron correlation.

To summarize, we have generalized the LDM method to adopt the nonorthonormal basis set and to include all the Coulomb differential overlap integrals. The generalized LDM method retains its linear scaling calculation nature for the excited state properties, which has been confirmed by the calculation of the absorption spectra of PA oligomers. With the employment of the nonorthonormal basis set and the inclusion of the complete differential overlap integrals, the generalized LDM method may be implemented readily at the $a b$ initio and semiempirical levels.

\section{ACKNOWLEDGMENTS}

Support from the Hong Kong Research Grant Council (RGC) and the Committee for Research and Conference Grants (CRCG) of the University of Hong Kong is gratefully acknowledged.

\section{APPENDIX: DERIVATION OF THE TDHF EQUATION IN NONORTHONORMAL BASIS}

In this Appendix, we outline the derivation of the EOM within the TDHF approximation for the reduced single electron density matrix in the nonorthonormal basis. We start with the definition of the reduced single electron density matrix $\rho$ in the spin-spatial representation,

$$
\begin{aligned}
\rho\left(\mathbf{r}_{1} \theta_{1}, \mathbf{r}_{1}^{\prime} \theta_{1}^{\prime}, t\right) \\
=N \int d \mathbf{r}_{2} d \theta_{2} d \mathbf{r}_{3} d \theta_{3} \cdots d \mathbf{r}_{N} d \theta_{N} \\
\quad \times \Phi\left(\mathbf{r}_{1} \theta_{1}, \mathbf{r}_{2} \theta_{2}, \ldots, \mathbf{r}_{N} \theta_{N}, t\right) \\
\quad \times \Phi^{*}\left(\mathbf{r}_{1}^{\prime} \theta_{1}^{\prime}, \mathbf{r}_{2} \theta_{2}, \ldots, \mathbf{r}_{N} \theta_{N}, t\right)
\end{aligned}
$$

$\Phi$ is the many-body wave function. Within the TDHF approximation, $\Phi$ may be expressed by a single Slater determinant

$$
\Phi\left(\mathbf{r}_{1} \theta_{1}, \mathbf{r}_{2} \theta_{2}, \ldots, \mathbf{r}_{N} \theta_{N}, t\right)=(N !)^{-\frac{1}{2}}\left|\begin{array}{cccc}
\psi_{1}\left(\mathbf{r}_{1} \theta_{1}, t\right) & \psi_{2}\left(\mathbf{r}_{1} \theta_{1}, t\right) & \cdots & \psi_{N}\left(\mathbf{r}_{1} \theta_{1}, t\right) \\
\psi_{1}\left(\mathbf{r}_{2} \theta_{2}, t\right) & \psi_{2}\left(\mathbf{r}_{2} \theta_{2}, t\right) & \cdots & \psi_{N}\left(\mathbf{r}_{2} \theta_{2}, t\right) \\
\vdots & \vdots & & \vdots \\
\psi_{1}\left(\mathbf{r}_{N} \theta_{N}, t\right) & \psi_{2}\left(\mathbf{r}_{N} \theta_{N}, t\right) & \cdots & \psi_{N}\left(\mathbf{r}_{N} \theta_{N}, t\right)
\end{array}\right|,
$$

where $\psi_{i}$ is the $i$ th occupied time-dependent molecular spinorbital and satisfies $\left\langle\psi_{i} \mid \psi_{j}\right\rangle=\delta_{i j}$. Integrating the rhs of Eq. (A1) results in

$$
\rho\left(\mathbf{r}_{1} \theta_{1}, \mathbf{r}_{1}^{\prime} \theta_{1}^{\prime}, t\right)=\sum_{k=1}^{N}\left|\psi_{k}\left(\mathbf{r}_{1} \theta_{1}, t\right)\right\rangle\left\langle\psi_{k}\left(\mathbf{r}_{1}^{\prime} \theta_{1}^{\prime}, t\right)\right| .
$$

The time derivative of Eq. (A3) may be expressed as

$$
\begin{aligned}
i \hbar \dot{\rho}\left(\mathbf{r}_{1} \theta_{1}, \mathbf{r}_{1}^{\prime} \theta_{1}^{\prime}, t\right)= & \sum_{k=1}^{N} i \hbar\left|\dot{\psi}_{k}\left(\mathbf{r}_{1} \theta_{1}, t\right)\right\rangle\left\langle\psi_{k}\left(\mathbf{r}_{1}^{\prime} \theta_{1}^{\prime}, t\right)\right| \\
& +\sum_{k=1}^{N} i \hbar\left|\psi_{k}\left(\mathbf{r}_{1} \theta_{1}, t\right)\right\rangle\left\langle\dot{\psi}_{k}\left(\mathbf{r}_{1}^{\prime} \theta_{1}^{\prime}, t\right)\right|
\end{aligned}
$$

The time evolution of the wave function $\Phi$ is determined by the Schrödinger equation

$$
H|\Phi\rangle=i \hbar \frac{\partial}{\partial t}|\Phi\rangle
$$

With the Frenkel principle, ${ }^{57}$ Eq. (A5) converts to

$$
\langle\delta \Phi|H| \Phi\rangle-\left\langle\delta \Phi\left|i \hbar \frac{\partial}{\partial t}\right| \Phi\right\rangle=0
$$

where $\delta \Phi$ is an arbitrary variation of $\Phi$. Since $\partial / \partial t$ behaves like a one-electron operator, we have

$$
\langle\delta \Phi \mid \dot{\Phi}\rangle=\sum_{i}\left(\left\langle\delta \psi_{i} \mid \dot{\psi}_{i}\right\rangle+\sum_{j(\neq i)}\left\langle\delta \psi_{i} \mid \psi_{i}\right\rangle\left\langle\psi_{j} \mid \dot{\psi}_{j}\right\rangle\right) .
$$

According to the Brillouin theorem, ${ }^{57}$ the first term of Eq. (A6) may be written as

$$
\langle\delta \Phi|H| \Phi\rangle=\sum_{i}\left\langle\delta \psi_{i}|\hat{F}| \psi_{i}\right\rangle
$$

Here $\hat{F}$ is the Fock operator corresponding to the Hamiltonian $H$,

$$
\hat{F}(t)=\hat{h}(t)+\hat{f}(t),
$$

where

$\hat{h}(t)=-\frac{1}{2} \nabla_{\mathbf{r}}^{2}+U(\mathbf{r})+\sum_{i}^{N}\left[\hat{J}_{i}(t)-\hat{K}_{i}(t)\right]$,

$\hat{f}(t)=e \mathbf{E}(t) \cdot \mathbf{r}$, 
$\hat{J}_{i}(t) \psi_{k}(\mathbf{r} \theta, t)$

$$
=\int d \mathbf{r}^{\prime} d \theta^{\prime}\left[\psi_{i}^{*}\left(\mathbf{r}^{\prime} \theta^{\prime}, t\right) \frac{1}{r_{12}} \psi_{i}\left(\mathbf{r}^{\prime} \theta^{\prime}, t\right)\right] \psi_{k}(\mathbf{r} \theta, t),
$$

$$
\begin{aligned}
& \hat{K}_{i}(t) \psi_{k}(\mathbf{r} \theta, t) \\
& \quad=\int d \mathbf{r}^{\prime} d \theta^{\prime}\left[\psi_{i}^{*}\left(\mathbf{r}^{\prime} \theta^{\prime}, t\right) \frac{1}{r_{12}} \psi_{k}\left(\mathbf{r}^{\prime} \theta^{\prime}, t\right)\right] \psi_{i}(\mathbf{r} \theta, t) .
\end{aligned}
$$

Assuming the electric field polarized along the chain axis $z$, $\hat{f}(t)=E(t) e \hat{z}$ with the dipole approximation. Substitution of Eqs. (A7) and (A8) in Eq. (A6) then gives

$$
\sum_{i}\left[\left\langle\delta \psi_{i}\left|\left(\hat{F}-i \hbar \frac{\partial}{\partial t}\right)\right| \psi_{i}\right\rangle-i \hbar \sum_{j(\neq i)}\left\langle\delta \psi_{i} \mid \psi_{i}\right\rangle\left\langle\psi_{j} \mid \dot{\psi}_{j}\right\rangle\right]=0 .
$$

Using the orthonormality constraint of the MOs, we have

$$
\begin{aligned}
& \left\langle\delta \psi_{i} \mid \psi_{i}\right\rangle+\left\langle\psi_{i} \mid \delta \psi_{i}\right\rangle=0, \\
& \left\langle\delta \psi_{i} \mid \psi_{j}\right\rangle=0, \quad(i \neq j) .
\end{aligned}
$$

We multiply Eqs. (A16) by an arbitrary constants $b_{j i}$, sum it over $i$ and $j$, and then subtract the resulting expression from Eq. (A14), and obtain

$$
\left(\hat{F}-i \hbar \frac{\partial}{\partial t}-i \hbar \sum_{j(\neq i)}\left\langle\psi_{j} \mid \dot{\psi}_{j}\right\rangle\right)\left|\psi_{i}\right\rangle-\sum_{j}\left|\psi_{j}\right\rangle b_{j i}=0 .
$$

Multiplying $\left\langle\psi_{k}\right|$ from the left and integrating Eq. (A17) for $k \neq i$, we find

$$
\left\langle\psi_{k}|\hat{F}| \psi_{i}\right\rangle-i \hbar\left\langle\psi_{k} \mid \dot{\psi}_{i}\right\rangle=b_{k i} .
$$

Similarly with $\left\langle\psi_{i}\right|$, we obtain

$$
\left\langle\psi_{i}|\hat{F}| \psi_{i}\right\rangle-i \hbar\left\langle\psi_{i} \mid \dot{\psi}_{i}\right\rangle-i \hbar \sum_{j(\neq i)}\left\langle\psi_{j} \mid \dot{\psi}_{j}\right\rangle=b_{i i} .
$$

On defining

$$
e_{j i}=\left\langle\psi_{j}|\hat{F}| \psi_{i}\right\rangle-i \hbar\left\langle\psi_{j} \mid \dot{\psi}_{i}\right\rangle \quad(\text { all } j, i),
$$

Eq. (A17) becomes

$$
\left(\hat{F}-i \hbar \frac{\partial}{\partial t}\right)\left|\psi_{i}\right\rangle=\sum_{j}\left|\psi_{j}\right\rangle e_{j i}
$$

which is the TDHF equation for $\left\{\psi_{j}\right\}$. It may be shown that $\left\{e_{i j}\right\}$ is a Hermitian matrix. According to Eq. (A21), Eq. (A4) is rewritten as $i \hbar \dot{\rho}\left(\mathbf{r}_{1} \theta_{1}, \mathbf{r}_{1}^{\prime} \theta_{1}^{\prime}, t\right)$

$$
\begin{aligned}
= & \sum_{k=1}^{N} \hat{F}\left|\psi_{k}\left(\mathbf{r}_{1} \theta_{1}, t\right)\right\rangle\left\langle\psi_{k}\left(\mathbf{r}_{1}^{\prime} \theta_{1}^{\prime}, t\right)\right| \\
& -\sum_{k, j=1}^{N}\left|\psi_{j}\left(\mathbf{r}_{1} \theta_{1}, t\right)\right\rangle e_{j k}\left\langle\psi_{k}\left(\mathbf{r}_{1}^{\prime} \theta_{1}^{\prime}, t\right)\right| \\
& -\sum_{k=1}^{N}\left|\psi_{k}\left(\mathbf{r}_{1} \theta_{1}, t\right)\right\rangle\left\langle\psi_{k}\left(\mathbf{r}_{1}^{\prime} \theta_{1}^{\prime}, t\right)\right| \hat{F} \\
& +\sum_{k, j=1}^{N}\left|\psi_{k}\left(\mathbf{r}_{1} \theta_{1}, t\right)\right\rangle e_{j k}\left\langle\psi_{j}\left(\mathbf{r}_{1}^{\prime} \theta_{1}^{\prime}, t\right)\right| \\
= & \hat{F}(t) \rho\left(\mathbf{r}_{1} \theta_{1}, \mathbf{r}_{1}^{\prime} \theta_{1}^{\prime}, t\right)-\rho\left(\mathbf{r}_{1} \theta_{1}, \mathbf{r}_{1}^{\prime} \theta_{1}^{\prime}, t\right) \hat{F}(t) .
\end{aligned}
$$

The occupied spin-spatial MO can be expanded in the spin-AO basis set,

$$
\psi_{k}(\mathbf{r} \theta, t)=\sum_{m} c_{m l}^{\sigma_{k}}(t) \chi_{m}(\mathbf{r}) \sigma_{k}(\theta),
$$

where $c_{m l}^{\sigma_{k}}$ is the coefficient which measures the amplitude of an electron at the AO $\chi_{m}$ for the $k$ th molecular spin-orbital $\psi_{k}, k \equiv\left(l, \sigma_{k}\right)$ with $l$ representing the spatial component of the $k$ th molecular spin-orbital, and $\sigma_{k}=\alpha$ or $\beta$ for its spin component. $\alpha(\beta)$ stands for spin up(down). Then the density matrix operator can be expressed in this basis set

$$
\rho\left(\mathbf{r} \theta, \mathbf{r}^{\prime} \theta^{\prime}, t\right)=\sum_{i j}\left|\chi_{i}(\mathbf{r})\right\rangle \rho_{i j}\left(\theta, \theta^{\prime}, t\right)\left\langle\chi_{j}\left(\mathbf{r}^{\prime}\right)\right|,
$$

see Eq. (16). After taking the time derivative and multiplying $\left\langle\chi_{m} \sigma\right|$ from the left and $\left|\chi_{n} \sigma\right\rangle$ from the right to Eq. (A24) and using Eq. (10), we have

$$
i \hbar\left\langle\chi_{m} \sigma\left|\dot{\rho}\left(\mathbf{r} \theta, \mathbf{r}^{\prime} \theta^{\prime}, t\right)\right| \chi_{n} \sigma\right\rangle==i \hbar \sum_{i j} S_{m i} \dot{\rho}_{i j}^{\sigma}(t) S_{j n}
$$

The matrix element of rhs of Eq. (A22) can be expressed as

$$
\begin{aligned}
\left\langle\chi_{m} \sigma\left|\left[\hat{F}, \rho\left(\mathbf{r} \theta, \mathbf{r}^{\prime} \theta^{\prime}, t\right)\right]\right| \chi_{n} \sigma\right\rangle & \\
= & \left\langle\chi_{m} \sigma\left|\left[\hat{F}, \sum_{k=1}^{N}\left|\psi_{k}(\mathbf{r} \theta, t)\right\rangle\left\langle\psi_{k}\left(\mathbf{r}^{\prime} \theta^{\prime}, t\right)\right|\right]\right| \chi_{n} \sigma\right\rangle \\
= & \sum_{l=\mathrm{occ}}\left\langle\chi_{m} \sigma|\hat{F}| \psi_{l}\right\rangle\left\langle\psi_{l} \mid \chi_{n} \sigma\right\rangle \\
& -\sum_{l=\mathrm{occ}}\left\langle\chi_{m} \sigma \mid \psi_{l}\right\rangle\left\langle\psi_{l}|\hat{F}| \chi_{n} \sigma\right\rangle \\
= & \sum_{l=\mathrm{occ}} \sum_{i j}\left[\left\langle\chi_{m}\left|\hat{F}^{\sigma}\right| \chi_{i}\right\rangle c_{i l}^{\sigma}\left(c_{j l}^{\sigma}\right) *\left\langle\chi_{j} \mid \chi_{n}\right\rangle\right. \\
& \left.-\left\langle\chi_{m} \mid \chi_{i}\right\rangle c_{i l}^{\sigma}\left(c_{j l}^{\sigma}\right) *\left\langle\chi_{j}\left|\hat{F}^{\sigma}\right| \chi_{n}\right\rangle\right] \\
= & \sum_{i j}\left(F_{m i}^{\sigma} \rho_{i j}^{\sigma} S_{j n}-S_{m i} \rho_{i j}^{\sigma} F_{j n}^{\sigma}\right) .
\end{aligned}
$$


In our Hamiltonian (1)-(4), the Fock matrix is given by $F_{k i}^{\sigma}=h_{k i}^{\sigma}+f_{k i}$, see Eqs. (18) and (19). By comparing Eqs. (A25) and (A26), we have the EOM (16).

${ }^{1}$ W. Yang, Phys. Rev. Lett. 66, 1438 (1991); W. Yang and T.-S. Lee, J. Chem. Phys. 103, 5674 (1995).

${ }^{2}$ P. Cortona, Phys. Rev. B 44, 8454 (1991).

${ }^{3}$ G. Galli and M. Parrinello, Phys. Rev. Lett. 69, 3547 (1992).

${ }^{4}$ S. Baroni and P. Giannozzi, Europhys. Lett. 17, 547 (1992).

${ }^{5}$ X.-P. Li, R. W. Nunes, and D. Vanderbilt, Phys. Rev. B 47, 10891 (1993).

${ }^{6}$ M. S. Daw, Phys. Rev. B 47, 10895 (1993).

${ }^{7}$ F. Mauri, G. Galli, and R. Car, Phys. Rev. B 47, 9973 (1993).

${ }^{8}$ P. Ordejón, D. A. Drabold, M. P. Grumbach, and R. M. Martin, Phys. Rev. B 48, 14646 (1993).

${ }^{9}$ W. Kohn, Chem. Phys. Lett. 208, 167 (1993).

${ }^{10}$ D. A. Drabold and O. F. Sankey, Phys. Rev. Lett. 70, 3631 (1993).

${ }^{11}$ W. Zhong, D. Tománek, and G. F. Bertsch, Solid State Commun. 86, 607 (1993).

${ }^{12}$ A. Gibson, R. Haydock, and J. P. LaFemina, Phys. Rev. B 47, 9229 (1993).

${ }^{13}$ M. Aoki, Phys. Rev. Lett. 71, 3842 (1993).

${ }^{14}$ E. B. Stechel, A. P. Williams, and P. J. Feibelman, Phys. Rev. B 49, 10088 (1994).

${ }^{15}$ S. Goedecker and L. Colombo, Phys. Rev. Lett. 73, 122 (1994).

${ }^{16}$ S.-Y. Qiu, C. Z. Wang, K. M. Ho, and C. T. Chan, J. Phys.: Condens. Matter 6, 9153 (1994).

${ }^{17}$ J. P. Stewart, Int. J. Quantum Chem. 58, 133 (1995).

${ }^{18}$ X. Chen, J.-M. Langlois, and W. A. Goddard, III, Phys. Rev. B 52, 2348 (1995).

${ }^{19}$ M. C. Strain, G. E. Scuseria, and M. J. Frisch, Science 271, 51 (1996).

${ }^{20}$ S. L. Dixon and K. M. Merz, Jr., J. Chem. Phys. 104, 6643 (1996).

${ }^{21}$ E. Schwegler, M. Challacombe, and M. Head-Gordon, J. Chem. Phys. 106, 9708 (1997).

${ }^{22}$ E. Hernández and M. J. Gillan, Phys. Rev. B 51, 10157 (1995).

${ }^{23}$ P. Ordejón, E. Artacho, and J. M. Soler, Phys. Rev. B 53, 10441 (1996).

${ }^{24}$ W. Kohn, Phys. Rev. Lett. 76, 3168 (1996).

${ }^{25}$ T. Iitaka, S. Nomura, H. Hirayama, X. Zhao, Y. Aoyagi, and T. Sugano, Phys. Rev. E 56, 1222 (1997).

${ }^{26}$ A. Takahashi and S. Mukamel, J. Chem. Phys. 100, 2366 (1994); G. H. Chen and S. Mukamel, J. Am. Chem. Soc. 117, 4945 (1995).

${ }^{27}$ P. Ring and P. Schuck, The Nuclear Many-Body Problem (Springer, New York, 1980).

${ }^{28}$ D. S. Chemla and J. Zyss, Nonlinear Optical Properties of Organic Molecules and Crystals (Academic, New York, 1987).

${ }^{29}$ A. Garito, R. F. Shi, and M. Wu, Phys. Today May, 51 (1994).
${ }^{30}$ D. C. Rodenberger, J. R. Heflin, and A. F. Garito, Nature (London) 359, 309 (1992).

${ }^{31}$ S. Etemad and Z. G. Soos, in Spectroscopy of Advanced Materials, edited by R. J. H. Clark and R. E. Hester (Wiley, New York, 1991), p. 87.

${ }^{32}$ Z. G. Soos, S. Ramesesha, D. S. Galvao, and S. Etemad, Phys. Rev. B 47, 1742 (1993)

${ }^{33}$ B. J. Orr and J. F. Ward, Mol. Phys. 20, 513 (1971).

${ }^{34}$ S. Tretiak, V. Chernyak, and S. Mukamel, J. Chem. Phys. 105, 8914 (1996).

${ }^{35}$ G. H. Chen and S. Mukamel, J. Phys. Chem. 100, 11080 (1996).

${ }^{36}$ S. Yokojima and G. H. Chen, Phys. Rev. B (submitted).

${ }^{37}$ S. Yokojima and G. H. Chen, Chem. Phys. Lett. 292, 379 (1998).

${ }^{38}$ R. Pariser and R. G. Parr, J. Chem. Phys. 21, 767 (1953); J. A. Pople, Trans. Faraday Soc. 49, 1375 (1953).

${ }^{39}$ A. A. Hansanein and M. W. Evans, Computational Methods in Quantum Chemistry, Quantum Chemistry Vol. 2 (World Scientific, Singapore, 1996).

${ }^{40}$ J. A. Pople, D. L. Beveridge, and P. A. Dobosh, J. Chem. Phys. 47, 2026 (1967).

${ }^{41}$ M. J. S. Dewar and W. Thiel, J. Am. Chem. Soc. 99, 4899 (1977).

${ }^{42}$ M. J. S. Dewar, E. G. Zoebisch, E. F. Healy, and J. J. P. Stewart, J. Am. Chem. Soc. 107, 3902 (1985).

${ }^{43}$ J. J. P. Stewart, J. Comput. Chem. 10, 209 (1989).

${ }^{44}$ X. Sun, Z. Shuai, K. Nasu, D. L. Lin, and T. F. George, Phys. Rev. B 44, 11042 (1991).

${ }^{45}$ J. A. Pople, D. F. Santry, and G. A. Segal, J. Chem. Phys. 43, S129 (1965).

${ }^{46}$ K. Ohno, Theor. Chim. Acta 2, 219 (1964).

${ }^{47}$ H. Zhao, S. Yokojima, X. Sun, and G. H. Chen (unpublished).

${ }^{48}$ R. W. Nunes and D. Vanderbilt, Phys. Rev. B 50, 17611 (1994).

${ }^{49}$ W. H. Press, B. P. Flannery, S. A. Teukolsky, and W. T. Vetterling, Numerical Recipes in C (Cambridge University Press, New York, 1988).

${ }^{50}$ G. H. Chen and S. Mukamel, Chem. Phys. Lett. 258, 589 (1996).

${ }^{51}$ G. H. Chen, Z. M. Su, S. Z. Wen, and Y. J. Yan, J. Chem. Phys. 109, 2565 (1998).

${ }^{52}$ W. Z. Liang and G. H. Chen (in preparation).

${ }^{53}$ L. Greegard, Science 265, 909 (1994); The Rapid Evaluation of Potential Fields in Particle Systems (MIT, Cambridge, MA, 1998).

${ }^{54}$ H.-Q. Ding, N. Karasawa, and W. A. Goddard, III, J. Chem. Phys. 97, 4309 (1992)

${ }^{55}$ S. Yokojima and G. H. Chen, Chem. Phys. Lett. (in press); S. Yokojima, D. H. Zhou, and G. H. Chen, Chem. Phys. Lett. (submitted).

${ }^{56}$ C. H. Martain and K. F. Freed, J. Chem. Phys. 101, 4011 (1994).

${ }^{57} \mathrm{R}$. McWeeny, Method of Molecular Quantum Mechanics, 2nd ed. (Academic, London, 1989). 